\title{
LETTER
}

\section{Sustained low-efficiency dialysis in surgical acute kidney injury - really useful?}

\author{
Matthias Heringlake*, Julika Schön and Hauke Paarmann \\ See related research by Schwenger et al., http://ccforum.com/content/16/4/R140
}

In a recent issue of Critical Care, we read with interest the article by Schwenger and colleagues [1] on sustained low-efficiency dialysis (SLED) versus continuous venovenous hemofiltration $(\mathrm{CVVH})$ in surgical patients with acute kidney injury. The authors have to be congratulated for their efforts to shed some light on the clinical usefulness of the SLED technology as an alternative to conventional renal replacement therapy (RRT) modalities. However, in our opinion, the study has some important limitations:

According to the protocol (NCT00322530), the authors aimed to enroll 100 patients per group, a sample size that may be justified only for an anticipated (but unlikely) mortality difference of roughly $20 \%$. Thus, the present study is clearly underpowered to detect differences in the primary outcome (90-day mortality).
Unfortunately, neither baseline pulmonary function nor indications for respiratory support are presented. This leaves ample room for speculation that - in such a small population with a high percentage of patients for whom severe pulmonary complications are common the shorter duration of ventilator support in the SLED group may be related to factors other than the RRT modality.

Despite the protocol (NCT00322530), the authors do not present renal recovery at 90 days. 'Time after RRT initiation' is a questionable renal outcome taking into account the lack of clearly defined RRT stop criteria and the unusual use of high-dose diuretics during (!) RRT.

With respect to these and other [2] limitations of the present study and the continuous RRT benefits shown in a recent meta-analysis [3], adequately powered studies comparing SLED and continuous venovenous hemodiafiltration are still urgently needed.

\section{Authors' response}

Vedat Schwenger, Markus Weigand and Christian Morath

We thank Heringlake and colleagues for their comments. Indeed, our study has several limitations that had been addressed thoroughly in the Limitations section of our article: 'the power of the study may be probably insufficient to finally judge whether one or the other treatment modality is superior in terms of survival' [1]. However, with comparable efficacy and safety of SLED and CVVH, the question comes down to economics, and the power of our study was clearly sufficient to discriminate differences in economics in favor of SLED therapy. Therefore, according to the best available evidence we have, dialysis techniques such as SLED are not inferior to continuous RRTs but do cost significantly less [1,4-6].

*Correspondence: heringlake@t-online.de

Department of Anesthesiology, University of Lübeck, Ratzeburger Allee 160, D - 23538 Lübeck, Germany
As discussed in the article, we feel (as Heringlake and colleagues do) that the shorter duration of ventilator support in the SLED group may be related to factors other than the RRT modality per se [1]. As discussed in the Limitations section, there are no generally accepted stop criteria for RRTs in intensive care units (ICUs). Since we had a surgical collective with often nonoliguric acute renal failure, we defined recovery of renal function as cessation of RRT but continuation of medical therapy.

In summary, to the best of our knowledge, we presented what is so far the largest prospective randomized trial for the comparison of SLED using a single-pass batch dialysis system (SLED-BD) and CVVH for the treatment of acute kidney injury in patients in the surgical ICU. Most outcome parameters were not different while SLED therapy was associated with significantly lower costs. 


\section{Abbreviations}

CVVH, continuous venovenous hemofiltration; ICU, intensive care unit; RRT, renal replacement therapy; SLED, sustained low-efficiency dialysis.

\section{Competing interests}

$\mathrm{MH}$ receives scientific support, consulting fees, and honoraria for lectures from Gambro Hospal (Gröbenzell, Germany). JS and HP declare that they have no competing interests. VS, MW, and CM have received lecture fees from Fresenius Medical Care (Bad Homburg, Germany). VS has received scientific grants from Gambro Corporate Research (Lund, Sweden). The study was funded by a grant from the European Nephrology and Dialysis Institute (Bad Homburg, Germany).

\section{Published: 22 January 2013}

\section{References}

1. Schwenger V, Weigand MA, Hoffmann O, Dikow R, Kihm LP, Seckinger J, Miftari N, Schaier M, Hofer S, Haar C, Nawroth PP, Zeier M, Martin E, Morath C: Sustained low efficiency dialysis using a single-pass batch system in acute kidney injury - a randomized interventional trial: the REnal Replacement Therapy Study in Intensive Care Unit Patients. Crit Care 2012, 16:R140.
2. Doig GS: Concerns regarding use of one-tailed tests in the SLED-BD vs. CVVH trial. Crit Care 2012, 16:448.

3. Schneider A, Bagshaw M, Glassford NJ, Bellomo R: Exposure to intermittent hemodialysis and renal recovery after acute kidney injury: a systematic review. Crit Care 2012, 16 (Suppl 1):P368.

4. Klarenbach S, Manns B, Pannu N, Clement FM, Wiebe N, Tonelli M: Economic evaluation of continuous renal replacement therapy in acute renal failure. Int J Technol Assess Health Care 2009, 25:331-338.

5. Srisawat N, Lawsin L, Uchino S, Bellomo R, Kellum JA: Cost of acute rena replacement therapy in the intensive care unit: results from The Beginning and Ending Supportive Therapy for the Kidney (BEST Kidney) study. Crit Care 2010, 14:R46.

6. Berbece AN, Richardson RM: Sustained low-efficiency dialysis in the ICU: cost, anticoagulation, and solute removal. Kidney Int 2006, 70:963-968.

doi:10.1186/cc11908

Cite this article as: Heringlake $M$, et al.: Sustained low-efficiency dialysis in surgical acute kidney injury - really useful? Critical Care 2013, 17:407. 